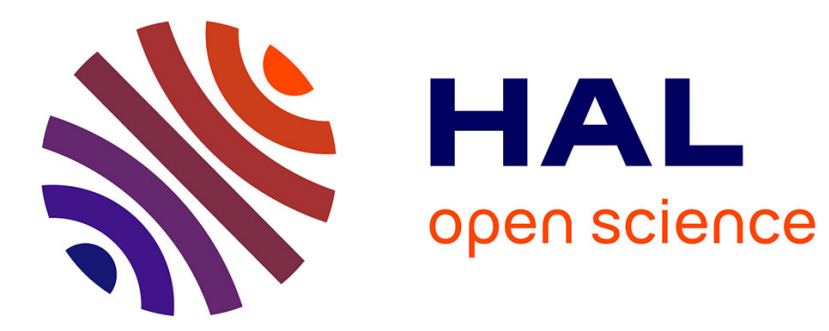

\title{
Czeżowski's axiological concepts as full-fledged modalities
}

\author{
Manuel Rebuschi
}

\section{To cite this version:}

Manuel Rebuschi. Czeżowski's axiological concepts as full-fledged modalities: We must either make what is good, or become revisionists. Forum Philosophicum, 2008, 13 (1), pp.103-110. hal-00351729

\section{HAL Id: hal-00351729 \\ https://hal.science/hal-00351729}

Submitted on 11 Jan 2009

HAL is a multi-disciplinary open access archive for the deposit and dissemination of scientific research documents, whether they are published or not. The documents may come from teaching and research institutions in France or abroad, or from public or private research centers.
L'archive ouverte pluridisciplinaire HAL, est destinée au dépôt et à la diffusion de documents scientifiques de niveau recherche, publiés ou non, émanant des établissements d'enseignement et de recherche français ou étrangers, des laboratoires publics ou privés. 


\title{
Czeżowski's axiological concepts as full-fledged modalities
}

\author{
We must either make what is good, or become revisionists
}

Manuel Rebuschi (manuel.rebuschi@univ-nancy2.fr)

Poincaré Archives, Nancy University

[The final version of this note is published in Forum Philosophicum, 13/1 (2008), 103-110]

\begin{abstract}
This short note provides a tentative formalization of Czeżowski's ideas about axiological concepts: Good and Evil are conceived of as modalities rather than as predicates. A natural account of the resulting "ethical logic" appears to be very close to standard deontic logic. If one does not resolve to become an antirealist regarding moral values, a possible way out is to become a revisionist about deontology: convert to intuitionism or other kind of revisionism for deontic logic, and remain classical for ethical logic.
\end{abstract}

Existence, goodness, and beauty, are not conceived in representations as are characters of objects, but are affirmed in judgments or evaluations.

Czeżowski 1953

Czeżowski provided an original conception of axiological concepts (or values) as objective and distinct from natural properties. They are modi essendi, i.e. modes acting on truths. For any sentence $p$, it can be good or not that it is true, as well as it can be necessary or not that it is true. [ ukasiewicz 2008] Hence the attribution of goodness to some fact or event should be like prefixing a sentence with some modal operator rather than applying a predicate to an (event-)entity. ${ }^{1}$

If one purports to analyze values, this is a good track to follow since it allows a specific neutrality regarding ontological issues: one could claim objectivism (or 'realism') about moral evaluation (in fact 'semantic realism': evaluations are objectively true or false) with no commitment to the existence of moral properties, coherently with some realistic account of natural properties. Supervenience of moral values upon natural properties is another nice upshot, together with the irreducibility of values to natural properties.

\section{Ethical logic}

Going into (smooth) formalization, classical propositional logic can be extended with a sentential operator G: $\mathrm{G} p$ being read " $p$ is good", or "it is good that $p$ ", where $p$ and other atomic letters denote assessable facts, events or states of affairs. ${ }^{2}$

$\mathrm{G}$ behaves like usual modalities, so that it can be handled using Kripke's possible-world semantics. We can consider an usual Kripke-model $\mathbf{M}=\langle\mathrm{W}, R, \mathrm{~V}\rangle$, where $\mathrm{W}$ is a nonempty set of possible worlds, $R$ an accessibility relation between worlds, and $\mathrm{V}$ a valuation function which devotes to each atomic formula the set of worlds where it is true. Starting from a given possible world $w$, another possible world $w^{\prime}$ is said to be morally accessible from $w$-i.e. $R w w^{\prime}$ - only if (what holds at) $w^{\prime}$ is not morally unacceptable from the standpoint of $w$. Then some fact (or event or state of affairs) $\varphi$ is good at $w$, if and only if $\varphi$ holds at every possible world morally accessible from $w$.

The standard modal system KD appears to be quite natural for such an operator, assuming that every tautology be considered as consistent with any good state of affairs: 
Axioms:

$$
\begin{array}{ll}
\mathrm{K} & -\mathrm{G}(\mathrm{p} \rightarrow \mathrm{q}) \rightarrow(\mathrm{Gp} \rightarrow \mathrm{Gq}) \\
\mathrm{D} & -\mathrm{Gp} \rightarrow \neg \mathrm{G} \neg \mathrm{p}
\end{array}
$$

Rules:

Taut. $\quad \mid-$ PL $\varphi \Rightarrow \mid-\varphi$

$[\mid-\mathrm{PL} \varphi$ meaning that $\varphi$ is a theorem of Propositional Logic (PL)]

RN $\quad-\varphi \Rightarrow \mid-\mathrm{G} \varphi$

Sub $\quad-\varphi \Rightarrow \mid-\varphi[\psi / \alpha]$

$[\varphi[\psi / \alpha]$ being the formula obtained from $\varphi$ substituting $\psi$ to

every token of the atom $\alpha$ in $\varphi]$

$\mathrm{G}$ being the analogue of the usual box operator of necessity ( $\square$ ), other modalities can be constructed in the following way:

$\begin{array}{ll}\text { " } \varphi \text { is evil" } & \mathrm{E} \varphi:=\mathrm{G} \neg \varphi \\ \text { " } \varphi \text { is morally acceptable" } & \mathrm{A} \varphi:=\neg \mathrm{E} \varphi\end{array}$

the latter being the analogue of the usual diamond operator of possibility $(\diamond)$.

Let us briefly indicate a few straightforward consequences of these definitions:

- $\quad$ Being evil $(\mathrm{E} \varphi)$ is not equivalent to not being good $(\neg \mathrm{G} \varphi)$.

- A fact or event $\varphi$ can be neither evil nor good, and still morally acceptable.

- $\quad$ Axiom D can be written using the operator $\mathrm{A}:-\mathrm{Gp} \rightarrow \mathrm{Ap}$, i.e. if $p$ is good, then $p$ is morally acceptable.

- $\quad$ Other axioms could be added, like

$$
\begin{array}{lll}
\text { o } & \text { Axiom 4: } \quad \mid-\mathrm{Gp} \rightarrow \mathrm{GGp} \\
\text { o } & \text { Axiom 5: } \mid-\neg \mathrm{Gp} \rightarrow \mathrm{G} \neg \mathrm{Gp} \text {, or equivalently: } \mid-\mathrm{AGp} \rightarrow \mathrm{Gp}
\end{array}
$$

- The usual Axiom T could not be added:

o Axiom T: $\quad \mid-\mathrm{Gp} \rightarrow \mathrm{p}$, i.e. everything which is good is the case.

Our 'good and evil logic', let us call it 'ethical logic,' (EL for short), is prima facie very close to standard deontic logic (SDL) (see [McNamara 2006] for a presentation of SDL): the operator E (resp. A) behaves exactly like $\mathrm{O}$ "it is obligatory that" (resp. $\mathrm{P}$ "it is permissible that"). We can even reinforce EL with an axiom taken from an usual strengthening of SDL:$$
+\mid-\mathrm{G}(\mathrm{Gp} \rightarrow \mathrm{p})
$$
EL+.

In the sequel, the systems based on the set of axioms $\{\mathrm{K}, \mathrm{D},+\}$ will be denoted as SDL + and

\section{Combining deontic logic with ethical logic}

Let us suppose that we have two distinct pairs of modalities: O/P and G/A, i.e. that the ethical operators are not reducible to the deontic ones. Such an assumption apparently fits with Czeżowski's metaethical classification according to which ethics is empirical, whereas deontology is normative. ${ }^{3}$ 
Both pairs of operators follow the KD+ axioms, i.e. we have a multi-modal system, with specific axioms relating the modalities. One axiom could be e.g.:

A1: $\quad \mid-\mathrm{O} p \rightarrow \mathrm{G} p$

Axiom A1 asserts the plausible deontic-ethical principle that if some action is obligatory, then it is good. ${ }^{4}$ By contraposition, $\mathrm{A} 1$ is equivalent to: $\mid-\neg \mathrm{G} p \rightarrow \neg \mathrm{O} p$, which means that if some fact is not good (maybe evil, maybe not), then it is not obligatory to realize it. This principle seems to be quite intuitive.

Another possible axiom would be the following one:

A2: $\quad \mid-\mathrm{G} p \rightarrow \mathrm{P} p$

Axiom A2 is weaker than the converse of A1. It states that if some fact is good, then it is permissible to realize it. By contrast, the converse of A1:

$\mathrm{A} 1 * \quad-\mathrm{G} p \rightarrow \mathrm{O} p$

means that if some fact is good, then it is obligatory to realize it. There are apparent reasons to rule out such a principle. For instance, one can consider two facts $p$ and $q$, both being good, the first one being the cause of the second one; then according to A2, one could produce $q$ only acting so that $p$ obtains, whereas with $\mathrm{A} 1 *$, one would be obliged to realize the two facts.

However, independently from A1, it seems that we are also committed to accept $\mathrm{A} 1 *$ : Let us assume SDL and EL, and suppose that $\mathrm{A} 1 *$ is false: there is a fact $p$ such that: $\mathrm{G} p \& \neg \mathrm{O} p$. But $\mathrm{G} p$ is equivalent to $\mathrm{E} \neg p$, and $\neg \mathrm{O} p$ is equivalent to $\mathrm{P} \neg p$. Then we get: $\mathrm{E} \neg p \& \mathrm{P} \neg p$. After a substitution of $q$ to $\neg p$, we obtain: $\mathrm{E} q \& \mathrm{P} q$. To put it in words: there is a fact $\mathrm{q}$ which is evil and permissible. This is obviously inadmissible.

So we should conclude that assuming SDL and EL implies assuming A1 and A1*. Now, A1 and $\mathrm{A} 1 *$ together mean that being good is equivalent to being obligatory.

This apparently contradicts Czeżowski's conception of two kinds of ethical values. We are left with two possible interpretations:

(1) Ethics is objective and, in some loose sense, ethical discourse is descriptive, but we have to realize everything that is objectively good; of course, as poor finite creatures, we cannot...

(2) Ethics as a whole is normative after all, and no ethical fact transcends our (practically manageable) duty.

\section{Comments on the upshot}

Is there any way out? As a matter of fact, several solutions seem available to save up Czeżowski's account.

(1) Axiom + is already strong: it is equivalent to $\mathrm{E}(\mathrm{G} p \wedge \neg p)$, which means that for any fact $p$, it is evil that $p$ is good and simultaneously does not hold. Anyway, the equivalence $\mathrm{G} p \leftrightarrow \mathrm{O} p$ is clearly independent from + .

(2) It can be objected to the conclusion that there is a confusion about the basic atomic sentences: do they denote (morally assessable) facts, or actions, or whatever else? Doesn't it have any effect on the expected relationship between operators $\mathrm{G}$ and $\mathrm{O}$ ? In one sense that would be true: some fact could be independent from any action and consequently morally assessable while not being "obligatory", "forbidden" or "permissible." Does it constitute a strong objection to the equivalence schema between $\mathrm{G} \varphi$ and $\mathrm{O} \varphi$ ?

No, it is rather a putting in perspective: the formation rules of EL as well as those of SDL should allow any formula to be prefixed by an operator. As a consequence, if we wish to combine 
the two systems, the set of atomic formulas of EL denoting atomic morally assessable facts must be constrained to (facts depending on) actions. Then the domain of objectively good facts could go far beyond that of obligatory facts. However, the equivalence between good and obligatory still concerns every fact reliant on action.

(3) One could object against the intuitions underlying the justification of A1*: it can be perfectly acceptable to contemplate the case of a fact $q$ which is both evil and permissible. For instance, denouncement can be considered as evil and (at least in some cases) permissible, e.g. when it is entailed by the forbidding of lying. Yet, admitting of such cases in the combination of ethical and deontological logics would lead us far from basic modal logics: we would have to enrich our formal language in order to regiment contexts of evaluation, so that an action be permissible in some given context and forbidden in another context. Ethical assessment might be absolute, whereas deontic judgment would be context-relative.

\section{Become revisionists!}

Another way out appears to be more faithful to Czeżowski's view: We can claim that ethical systems account for a normative background, and that deontic systems account for what has to be done in front of this background.

According to Czeżowski, ethical ("axiological" in Czeżowski's terminology) norms are inductively generalized from basic moral judgments, and they do constitute ethical ("axiological") laws about what is good and what is evil. Deontic norms which state what is obligatory, what is permitted and what is forbidden, are a priori and cannot be reduced to ethical norms - some of the deontic norms regiment interpersonal obligation, where ethic ("axiological") norms only regiment individualistic ethics. Nevertheless, we need deontic norms to make ethical laws effective since deontic laws determine the way moral agents should behave. Focusing on individualistic ethics, ethical laws and deontic laws seemingly coincide.

However, it still seems absurd to consider that a moral agent is expected to perform every action which is evaluated to be good. For instance, it can be assumed to be good that I give 100 euros to my sister $\left(p_{1}\right)$, as well as it can be good that I give her 200 euros $\left(p_{2}\right)$; yet I would not be expected to perform each of these possible good actions, but at most one of them. We thus would stick to axiom A1, but get rid of A1*.

How can this be regimented in ethical and deontic logic? A natural solution is to adopt $a$ revisionist strategy: abandon classical logic and implement some constructivist restriction of it for the deontic operators, while sticking to classical logic for the ethical operators. There are many versions of restrictions of classical logic - starting from the historical intuitionist case to more recent substructural logics, connected to more or less radical accounts of anti-realism. The common idea of these revisionist strategies is to restrict the inference rules so that the set of logical consequences of a set of axioms gets smaller. So an ethical system would constitute a (classical) frame at the background consisting in all the good laws, and the deontic system would yield a proper subset of the good laws as the set of obligatory actions.

Let us give an example with an intuitionism. So we consider a classical ethical system together with an intuitionist deontic system. Here A1 obviously holds, but A1* does no more: like in the above argument, let us suppose that $\mathrm{A} 1 *$ is false, i.e. that there is some fact $p$ which is good $(\mathrm{G} p)$ but not obligatory ( $\neg \mathrm{O} p$ ). After a substitution of $q$ to $\neg p$, we obtain that there is a fact which is evil $(\mathrm{E} q)$, while its negation is not obligatory $(\neg \mathrm{O} \neg q)$. Now, as the deontic operator is no more classical, one cannot infer from $(\neg \mathrm{O} \neg q)$ that $\mathrm{P} q$, i.e. that there is a fact $q$ which is simultaneously evil and permissible: the counterintuitive conclusion reached above with classical deontic logic is blocked. 
So we might conclude that the prima facie equivalence between ethical and deontic systems is avoidable provided one rejects classical logic for deontic systems; with a restricted logical system, one can infer less consequences from deontic systems than from ethical systems. So the systems will no more coincide.

\section{Further issues}

I have sketched an illustration of the promising usefulness of a formalization of ethics using standard modal logics.

Other combinations could be conceived of. One can combine EL with modal epistemic logic

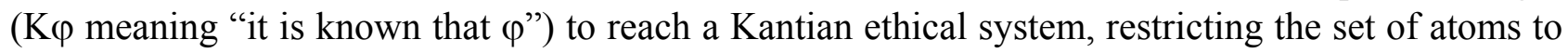
actions (involving intention), and adding a specific axiom schema relating K to G: $\mathrm{G} \varphi \rightarrow \mathrm{KG} \varphi$. Since knowledge is usually considered as factive $(\mathrm{K} \varphi \rightarrow \varphi)$ it would imply a collapse of modalities: $\mathrm{G} \varphi \leftrightarrow \mathrm{KG} \varphi$, i.e. being good is being known to be good.

Ethical logic can be combined with usual modalities $(\square$ and $\diamond)$ interpreted as metaphysical modalities. Philosophical issues similar to those between Descartes and Leibniz about eternal truths could be formulated in such a frame: would one assume that: $\mathrm{G} \varphi \rightarrow \square \mathrm{G} \varphi$ ?

A fundamental issue to address for ethical logic is the following: Shouldn't we revise EL to reject some of its theorems? For instance, shall we admit of the following theorem:

$* \mid-\mathrm{G}(\mathrm{p} \& \mathrm{q}) \rightarrow(\mathrm{Gp} \& \mathrm{Gq})$

It seems intuitively that some joint events can be assessed as good, whereas considering them separately would urge a shift of evaluation. But if we want to reject $\left(^{*}\right)$, it would lead ethical logic to non-normal systems, with certainly important new difficulties. ${ }^{6}$

\section{References}

Czeżowski, T. (1949). "Ethics as an Empirical Science." Philosophy and Phenomenological Research, 14, (2) (Dec., 1953), 163-171.

Gawor, L., \& Zdybel, L. (2005). “Elements Of Twentieth Century Polish Ethics.” In Jedynak S. (Ed.): Polish Axiology: The $20^{\text {th }}$ Century and beyond, Polish Philosophical Studies, V. Cultural Heritage and Contemporary Change, Series IVA, Central and Eastern Europe, Volume 25. [URL $=<$ http://www.crvp.org/book/Series04/IVA25/contents.htm>]

Łukasiewicz, D. (2008). “Metaphysics of Axiological Realism.” Philosophia Scientice, 12 (1), 2008, 57-74.

McNamara, P. (2006). “Deontic Logic.” In Zalta E. N. (Ed.), The Stanford Encyclopedia of Philosophy (Spring 2006 Edition). [URL $=<$ http://plato.stanford.edu/archives/spr2006/entries/logic-deontic/ $>$ ].

\footnotetext{
Notes

1 "In all these examples there occurs a sentence composed of modus and dictum (if we use the classical terminology); modus is the expression: 'It is necessary that...', 'It is true that...' etc., dictum is the sentence following modus. Today we call modus a sentential functor. The circumstance that modal functors (necessary, possible), the functor of assertion (it is true that...) and the functor of evaluation (good, beautiful) do require as their complement a sentence (and not a name, as other adjectives do when they play the role of an attribute) shows that these modi cannot be given in presentations but that they are asserted by propositions. Anyway, it has been well known for a long time - Hume and Kant were conscious of it - that they (modi) cannot be given in any presentation, and even that these expressions are 'contentless'; they express only someone's reaction to a certain state of affairs." Czeżowski 1965, Filozofia na rozdrożu, Warszawa: PWN, 38-39. Quoted by [Łukasiewicz 2008]

${ }^{2}$ These are not equivalent notions and a uniform choice should be made regarding the interpretation of atomic letters. Be that as it may, I will leave this question unsolved since it does not seem to have any consequence for the formalization put forward.
} 
3 "From a meta-ethical point of view Czeżowski classified all ethical systems into two categories, axiological and deontological. The first category, based on direct experience of moral values, strives towards inductive generalisation of the principles of moral behaviour (for example, requiring truthfulness or demanding that courage be combined with caution). Deontological ethics, on the other hand, defines moral obligations, justifying them by means of universal moral axioms accessible to intuition (e.g. in the form of Kant's categorical imperative, evangelical love of one's neighbour or the principle of common kindness)." [Gawor \& Zdybel 2005] - See also [Czeżowski 1953] about the empirical character of ethics.

${ }^{4}$ The axiom is intended to be interpreted with a moral meaning, but it should be noticed that it would be acceptable adopting a medical or any other meaning - the requirement being that the interpretation be uniform for the two operators.

${ }^{5}$ In intuitionist modal logic, $\square$ and $\diamond$ are no more mutually definable: the schema " $\neg \neg \varphi \rightarrow \diamond \varphi$ " is not intuitionistically valid.

${ }^{6}$ I wish to thank Philippe Nabonnand, Fabien Schang, Tero Tulenheimo, and especially Darek Łukasiewicz for their enlightening comments and suggestions on an earlier version of this note. 